PROFESIONALES Y HERRAMIENTAS PARA EL DESARROLLO LOCAL Y SUS SINERGIAS TERRITORIALES. EVALUACIÓN Y PROPUESTAS DE FUTURO IX Coloquio Nacional de Desarrollo Local del GTDL-AGE 

ANTONIO MARTÍNEZ PUCHE, XAVIER AMAT MONTESINOS, ISABEL SANCHO CARBONELL y DANIEL SANCHIZ CASTAÑO (EDS.)

\section{PROFESIONALES Y HERRAMIENTAS PARA EL DESARROLLO LOCAL Y SUS SINERGIAS TERRITORIALES. EVALUACIÓN Y PROPUESTAS DE FUTURO}

IX Coloquio Nacional de Desarrollo Local del GTDL-AGE

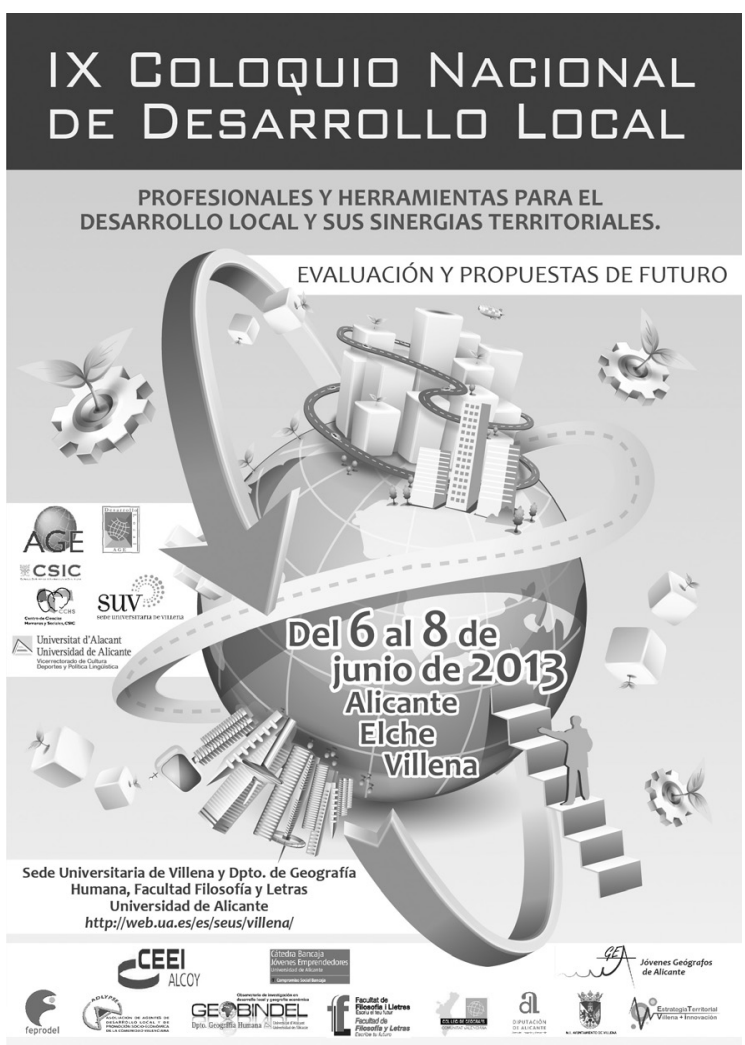


Este libro ha sido examinado y valorado por evaluadores ajenos a la Universidad de Alicante, con el fin de garantizar la calidad científica del mismo.

Publicacions de la Universitat d'Alacant

03690 Sant Vicent del Raspeig

Publicaciones@ua.es

http://publicaciones.ua.es

Telèfon: 965903480

(C) Antonio Martínez Puche, Xavier Amat Montesinos,

Isabel Sancho Carbonell y Daniel Sanchiz Castaño (eds.), 2016

(C) d'aquesta edició: Universitat d'Alacant

ISBN: 978-84-16724-00-0

Dipòsit legal: A 92-2016

Disseny de coberta: candela ink

Composició: Página Maestra (Miguel Ángel Sánchez Hernández)

Impressió i enquadernació: Guada Impresores

\section{unte \\ Unión de Editoriales
Universitarias Españolas \\ WWW.une.es
WWA}

Esta editorial es miembro de la UNE, cosa que garantiza la difusión y comercialización nacional y internacional de sus publicaciones.

Reservados todos los derechos. Cualquier forma de reproducción, distribución, comunicación pública o transformación de esta obra sólo puede ser realizada con la autorización de sus titulares, salvo excepción prevista por la ley. Diríjase a CEDRO (Centro Español de Derechos Repográficos, www.cedro.org) si necesita fotocopias o escanear algún fragmento de esta obra. 


\title{
EL POLO DE COMPETITIVIDAD COMO MESA DE COLABORACIÓN PÚBLICO-PRIVADA
}

\author{
Igone Porto \\ Behargintza Leioa \\ Iporto001@ikasle.ehu.es \\ José Ramón Otegi \\ Escuela Superior de Ingeniería del País Vasco \\ Joserra.otegi@ehu.es
}

RESUMEN

Este caso de desarrollo territorial se sustenta en 2 claves: Por un lado, el Partenariado Público Privado, que establece una mesa con diferentes perspectivas de una comarca industrial. Por otro lado, el modelo desarrollado se ha impulsado desde la perspectiva privada de base, bottom up, buscando las adhesiones al proyecto de diferentes niveles públicos. Debe destacarse el hecho de que este caso está ligado con un nivel de cohesión social elevado.

Palabras Clave: Partenariado Público-Privado, Relaciones empresariales, Bottom Up, Capital Social.

THE COMPETITIVENESS POLE AS A PUBLIC PRIVATE PARTNERSHIP

\section{ABSTRACT}

Two keys address this case of regional development. On one hand, the Public Private Partnership establishes different perspectives of an industrial county. On the other hand, the developed model has been driven bottom up, from the private perspective, seeking adhesions to the project at different public levels. It should be noted that this case is linked to high level of social cohesion.

Key words: Public Private Partnership, Business relationships, Bottom Up, Social Capital. 


\section{INTRODUCCIÓN}

Desde el año 2006, y a instancias de la Asociación de Empresas del Duranguesado, AED, en colaboración con actores público-privados de la comarca, se inicia un proyecto de fomento de la competitividad territorial, bajo la marca Polo de Competitividad del Duranguesado. Este se articula como una red informal sin estructura jurídica, que a través de la cooperación de dichos actores busca desarrollar proyectos tecnológicos innovadores en el ámbito de la comarca.

Este objetivo pretendido por la red de actores busca el fortalecimiento tecnológico de esas empresas, y por consiguiente, un beneficio individual y colectivo para los agentes del entorno:

1. Optimización tecnológica y diversificación del conjunto productivo.

2. Intercambio de conocimientos y experiencias, como medidas de difusión de conocimiento tácito.

3. Imagen de marca del Duranguesado a nivel industrial en el exterior.

Establecimiento de alianzas y convenios de colaboración con actores externos, de potencial enriquecimiento del entorno.

El objetivo general de la investigación que aquí se presenta, es el análisis de los actores pertenecientes a esa red informal, y la valoración de los mismos por parte de las empresas de la Comarca.

\section{Presentación del caso. Actores del entorno}

El Duranguesado es una comarca situada en la región oriental de Bizkaia que limita con Gipuzkoa al Este y Araba al Sur. Su población es de 76.365 habitantes sobre una superficie de $291,04 \mathrm{Km}^{2}$ con una densidad de población media de 262,39 Hab/ $\mathrm{Km}^{2}$.

Considerando la importancia de la formación del capital humano dentro de los procesos formativos, y productivos de un entorno (Cañibano, 2005), se presenta a continuación la relación entre niveles formativos en los tres niveles administrativos cercanos al entorno.

Tabla 1. Comparativa de Población y Nivel de Estudios

\begin{tabular}{|l|c|c|c|}
\hline \multicolumn{1}{|c|}{ Estudios } & Duranguesado & Bizkaia & CAPV \\
\hline Secundarios & $19,16 \%$ & $21,40 \%$ & $21,64 \%$ \\
\hline Formación Profesional & $17,26 \%$ & $14,69 \%$ & $15,29 \%$ \\
\hline Titulación Universitaria Media & $8,02 \%$ & $8,57 \%$ & $8,56 \%$ \\
\hline Titulación Universitaria Superior & $11,30 \%$ & $15,64 \%$ & $14,72 \%$ \\
\hline
\end{tabular}

Fuente: Eustat, 2010. 
Destaca un porcentaje superior, 2,5\%, en los titulados de Formación Profesional del Duranguesado respecto a Bizkaia, y del 2\% respecto al País Vasco. Por otro lado, existe un $2 \%$ menor de personal no titulado. Según las entrevistas cualitativas realizadas a actores del entorno este hecho se corresponde con, el marcado carácter industrial de la comarca, con necesidades concretas de ámbito productivo.

\subsection{Tejido Productivo: Empresas}

El 92\% de las actividades desempeñadas en la comarca están vinculadas con el sector metal-mecánico, bien desde la pertenencia a las tecnologías principales de fabricación -Estampación (14,95\%), Fundición (12,75\%), o Forja $(5,71 \%)$ - o bien desde la disposición de servicios complementarios -Mecanizado, Tratamiento, Calderería o Soldadura, etc-.

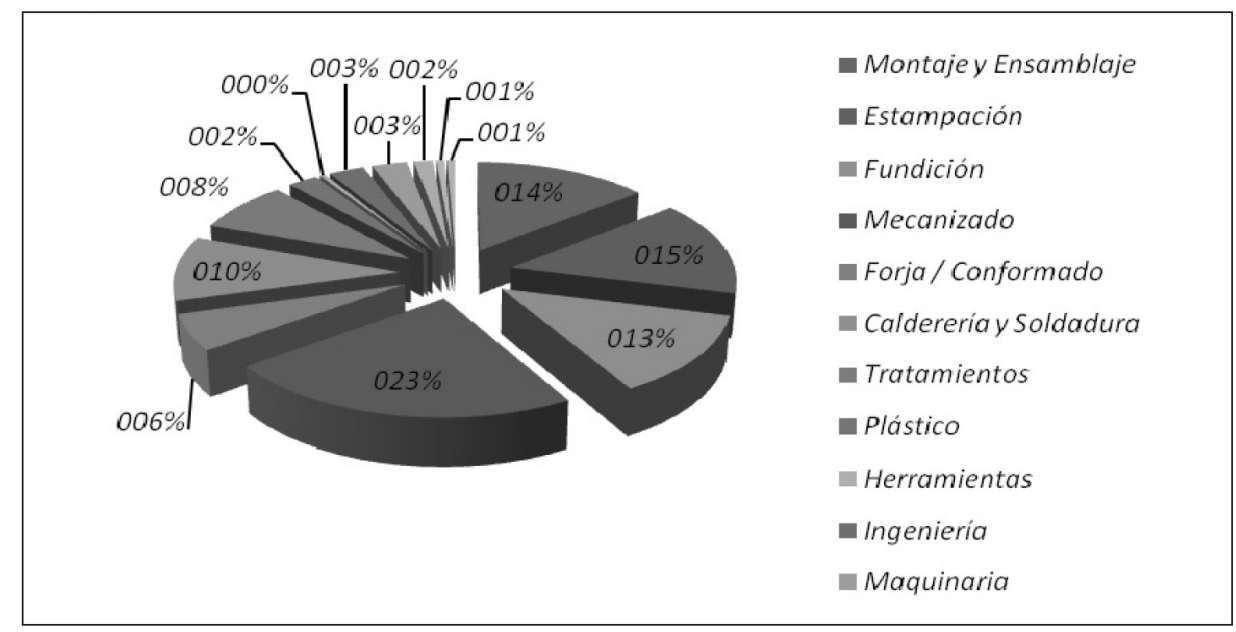

Gráfica 1. Tejido Productivo de la comarca según líneas de actividad

Fuente: Elaboración Propia.

Se identifica una cadena de valor, basada en servicios complementarios entre las diferentes tecnologías del entorno, sin producto propio, orientada a servicios de manufactura a clientes externos en la comarca.

Se puede caracterizar la industria del entorno de la siguiente manera:

1. Se constata amplio conocimiento y tradición industrial alrededor de la industria manufacturera del metal.

2. Destaca el tejido micro-económico industrial, con un $90 \%$ de pequeñas empresas, de tamaño pequeño o micro.

3. Resalta la escasa tracción tecnológica - innovadora de las empresas grandes. Esto es, las empresas grandes no tienen relaciones de coope- 
ración con las empresas del entorno con las que mantienen relaciones productivas.

4. El 92\% de los actores tienen como mercado primario a la Automoción, y como secundario en un $45 \%$ Energía y Máquina Herramienta.

5. Nivel tecnológico medio-bajo. Adaptación a nuevos procesos y requerimientos del mercado, pero la incorporación de nuevas tecnologías relacionadas con sus procesos clave, no se produce con la intensidad que el mercado requiere.

6. Las empresas de la comarca son subcontratistas, sin producto finalista respecto al cliente final, lo cual ocasiona una gran dependencia de sus clientes limitando las posibilidades de desarrollo de la propia empresa.

7. Escaso conocimiento de las actividades que desarrollan el resto de las empresas del Duranguesado - desconocimiento de la cadena de valor y del mapa industrial-, que unido a la histórica poca cultura de cooperación entre empresas, provoca que la competitividad de las empresas y las oportunidades se reduzcan.

De cara a tener una voz unitaria con otros agentes, se estableció una Asociación de Empresas del Duranguesado, AED, que busca fortalecer la situación de las empresas de la comarca.

\subsection{Centros de Conocimiento}

En el entorno se cuenta con dos centros de Formación Profesional, que si bien tienen un papel principal de formación primaria, mantienen vinculación estrecha con el tejido productivo, dedicando esfuerzos a la formación de los recursos humanos ya activos en el mercado laboral, así como a la labores de adaptación y difusión tecnológica, a través de las mejores prácticas en cada ámbito. Otro hecho destacable es la disposición de los profesores de estos centros a la colaboración directa con las empresas del entorno mostrándose abiertos a la participación en proyectos prácticos que optimicen los procesos productivos.

A su vez, se cuenta en el entorno con un Centro Tecnológico privado, AZTERLAN, perteneciente a la corporación tecnológica IK4. Cuenta con más de 30 años de experiencia acreditada como Centro Sectorial de Investigación en Metalurgia.

Es un centro cercano a las empresas, orientado al desarrollo de investigación aplicada. El 20\% de los clientes del centro se corresponden con empresas de la comarca. Se erige en actor que mantiene una estrecha colaboración con universidades y centros tecnológicos europeos, como vías de apertura tecnológica a conocimiento exterior. Además de la actividad de $\mathrm{I}+\mathrm{D}$, cuenta con un departamento de Materiales que da servicio a las necesidades de las empresas, ofertando un amplio espectro de análisis y ensayos para una completa caracterización de sus productos. A partir de estos servicios, se establecen relaciones 
de confianza con las empresas, lo que permite plantear proyectos de optimización de productos o procesos, incrementando de esta forma la innovación de las mismas.

Se encuentra también en el entorno un centro de investigación e innovación abierta, Automotive Intelligent Center AIC, vinculado con el sector de Automoción, donde las empresas buscan la mejora competitiva a través de la cooperación. Es un centro promovido por la colaboración entre Empresas, Centros Tecnológicos y la Administración foral, y está gestionado por la Asociación Clúster de Automoción ACICAE, con sede en la comarca.

Sin embargo, las empresas no identifican con la misma cercanía a AIC en comparación con AZTERLAN. No existe una gran permeabilidad entre este centro, sus investigaciones y los desarrollos de interés para las empresas que conforman el tejido productivo del entorno.

\subsection{Agentes Públicos}

La escasez presupuestaria de los municipios que conforman el entorno y la necesidad de dar respuesta al tejido productivo desde el ámbito más cercano, entre otros, lleva a tender puentes entre las corporaciones locales, de cara a la agrupación a nivel de valle. Esto llevó el 13 de Febrero de 1971 a erigir la Mancomunidad de la Merindad de Durango, de conformidad con lo previsto en la Ley de Régimen Local.

Los servicios que se prestan desde este ente público son de diversa índole, encargándose la Agencia de Desarrollo Local y Promoción económica, Behargintza, del trato y vinculación con empresas. Behargintza, como oficina de promoción económica de ámbito comarcal, centra sus actividades en tres líneas principales: Atención al desempleado e Intermediación Laboral, Emprendizaje y Promoción y optimización del tejido empresarial.

De cara a ayudar al tejido productivo, la Agencia desarrolla las siguientes acciones:

- Asesoramiento en materia de Ayudas y Subvenciones. Colaboración en su solicitud.

- Jornadas formativas en áreas de interés legal, financiero o de promoción.

- Desarrollo de una Guía Virtual Empresarial, para identificación de los agentes activos en el entorno y su función.

- Servicio de Asesoramiento Técnico y Jurídico en materia ambiental.

Si bien la competencia legislativa en el ámbito de promoción económica, corresponde al Gobierno Vasco, se considera de interés la disposición de una agencia de ámbito comarcal, para acompañar desde el terreno a las empresas ubicadas en el entorno, y facilitar así, su interlocución con instancias superiores.

Dentro del proyecto Polo de Competitividad se ha buscado la participación activa de este actor, así como la relación con agencias de ámbitos foral y auto- 
nómico. Esta relación coincide con uno de los objetivos establecidos en el Plan de Competitividad, Tecnología e Innovación 2015 (PCTI, 2012) del Gobierno Vasco, a través de la articulación de redes de colaboración entre los actores públicos multinivel.

\subsection{Capital Social}

Un elemento difícil de medir, que permite la cohesión entre los actores, es el Capital Social (Putnam, 2001), entendiendo este como las redes sociales horizontales y verticales entre las personas, en base a normas y patrones culturales, que impactan en la productividad y la competitividad, al facilitar estos elementos la coordinación y la cooperación como base para el bien común del entorno.

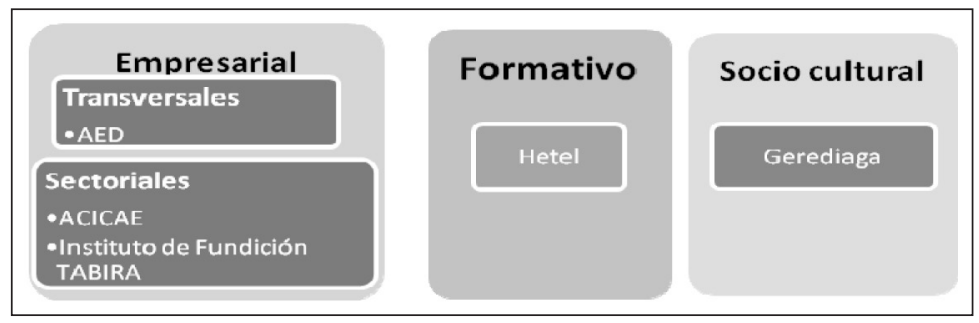

Imagen 1. Asociaciones activas del entorno.

Fuente: Elaboración Propia.

El entorno dispone de una cultura propia que se traslada también al entorno empresarial. El estudio cuantitativo permite confirmar este hecho, como demuestra la valoración por parte del $79 \%$ de las empresas encuestadas, sobre la potencialidad industrial de la comarca, con un carácter endógeno para la creación de competitividad territorial.

\subsubsection{El rol de los Agentes de Confianza}

Un hecho destacable dentro del modelo de desarrollo local gestado en el entorno, reside en la aparición de la figura de Agentes de proximidad o de confianza. Esta figura asimilable a lo que Eriksson llama en su modelo de sistema regional de innovación, Dinamizadores (Eriksson, 2000), no ha sido identificada en otros casos de desarrollo territorial con la misma fortaleza que la identificada en la Comarca.

Concretamente, los Agentes de Proximidad son actores pertenecientes al entorno, que por su conocimiento del mismo desde una perspectiva social y empresarial, consiguen que las empresas de la comarca confíen en ellos para la realización de Diagnósticos Empresariales individuales. A partir de estos 
diagnósticos, se identifican proyectos individuales de optimización tecnológica, así como proyectos en cooperación con otros actores de la comarca.

Los Agentes de Confianza corresponden con los siguientes perfiles:

- Profesores de Centros de Formación Profesional.

- Investigadores del Centro Tecnológico principal de la Comarca.

- Agentes Productivos: Personas jubiladas de empresas activas en la Asociación de empresas de la comarca o reconocidas por su bagaje.

El rol de Agentes de Confianza, no es posible solo gracias a una mentalidad empresarial común, sino a la disposición de unos valores comunes, que facilitan las relaciones de confianza, formales e informales, tejidas a lo largo del tiempo. Es a través de estos valores y cultura, que aumenta el Capital Social.

\subsection{Red del Polo de Competitividad}

La red de actores que conforman el grupo tractor del Polo de Competitividad esta conformada por los siguientes:

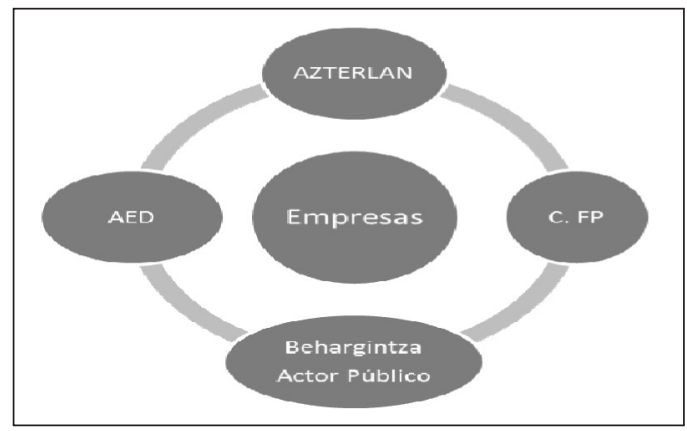

Imagen 2. Red de actores del Polo de Competitividad.

Fuente: Elaboración Propia.

\section{DEFINICIÓN DE LAS RELACIONES EXISTENTES: EMPRESARIALES Y SOCIALES}

Se pretende recoger en este apartado la vinculación entre las empresas del sector metal-mecánico existentes en la comarca. Estas vinculaciones conformarían la Cadena de Valor Territorial (Trejo Téllez, 2011, Albors et Hidalgo, 2012) existente en el territorio, desde una perspectiva de clientes a partir del rol de los proveedores disponibles.

\subsection{Relaciones Formales}

Se han medido las redes reales de relación en función del grado de colaboración entre las empresas. El análisis de las relaciones permite describir un tejido empresarial con relaciones predominantemente al albor de la cadena de valor, esto 
es, dentro de cadenas de suministro. Esto coincide con la disposición de lazos empresariales en un entorno maduro, que como identifica Otero (Otero, 2010), producen innovaciones en proceso, dentro de las cadenas de valor propias de los sectores para los que operan las empresas implicadas.

En función de la complementariedad de las tecnologías principales de transformación metal-mecánica, se identifican las siguientes redes de colaboración de suministro:

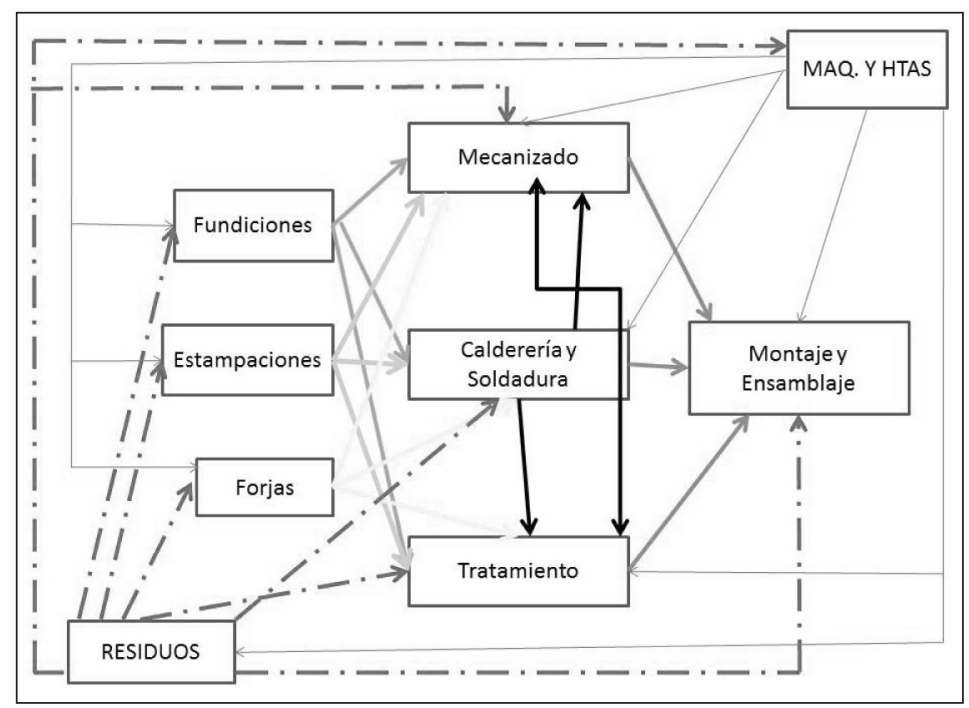

Imagen 3. Cadena de valor metal-mecánica de la comarca.

Fuente: Elaboración Propia.

El análisis de las diferentes cadenas de colaboración de la comarca, permite identificar dos tipos de cadenas. Las cadenas de suministro, aproximadamente un $85 \%$ de las identificadas -con un $45 \%$ de subcontratación total y relaciones cliente proveedor y un $40 \%$ con colaboración en producción-, y las cadenas de colaboración, en $\mathrm{I}+\mathrm{D}+\mathrm{i}$, en un $15 \%$. De este dato, un $5 \%$ correspondería con cadenas de integración total y apertura entre los actores del entorno. Este hecho, en el que predominan las relaciones de suministro pone de relieve una red de relaciones de suministro tupidas que caracterizan al tejido empresarial del Duranguesado. Esta característica se considera una fortaleza para la definición de futuras actividades de desarrollo de la comarca.

Además de las relaciones formales entre actores empresariales, se producen otras relaciones con otro tipo de actores del entorno, como son los centros de conocimiento, asociaciones sectoriales, tecnológicas etc. o las instituciones públicas. No obstante, las relaciones entre los actores productivos, clientes- 
proveedores, son las prioritarias dentro del sistema, y serán los actores clave para la gestación de un entorno industrial innovador.

Para medir las relaciones entre las empresas con el resto de actores del tejido, se parte de los valores de un estudio desarrollado por el Centro Tecnológico TEKNIKER en el año 2011. Este estudio analiza el grado de cooperación entre las empresas con todos los actores disponibles en la comarca, así como posibles nexos con actores del exterior:

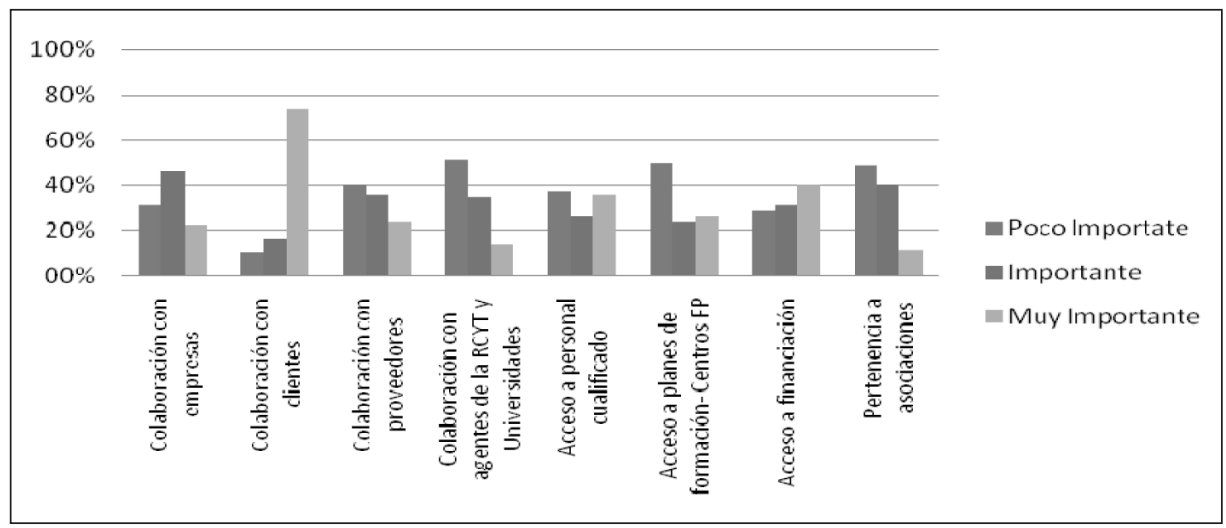

Gráfica 2. Valoración de las relaciones con actores del terreno por parte de empresas.

Fuente: TEKNIKER, 2011.

Dentro de este estudio, se identifican como actores prioritarios para la cooperación por parte de las empresas a los Clientes (70\%), seguidos del ámbito de Capital humano (38\%), y Centros de Formación Profesional (24\%). Se considera irrelevante la vinculación con Centros tecnológicos y universidades $(12 \%)$ o la pertenencia y relaciones de cooperación con asociaciones (10\%).

\subsection{Relaciones Informales}

Las relaciones informales (Mohannak, 1998) se encuentran influenciadas no solo por las relaciones empresariales sostenidas en el tiempo que conlleven relaciones de amistad, sino también por hechos socio-culturales ajenos a la actividad empresarial como tal. A este respecto, se consideran:

- Los lazos familiares y de amistad.

- Lazos de vecindad o Proximidad geográfica-espacial.

- Eventos socio-culturales: Actividades de ocio que se comparten.

No se dispone en esta investigación información detallada sobre el origen y pertenencia de las personas, de cara a poder analizar la razón de las relaciones informales en el tejido productivo e industrial. La impresión mayoritaria en las entrevistas cualitativas ha apuntado que las personas del Duranguesado 
se quedan a trabajar en la Comarca en un alto índice, pero no se disponen de información que confirme este hecho.

\section{ACTORES QUE INFLUYEN EN LA COOPERACIÓN}

A partir de los actores relevantes para las empresas, se ha considerado de interés analizar el grado de confianza respecto a los actores concretos del entorno, de cara a validar la configuración de agentes que integran el comité del Polo de Competitividad como estructura válida para la dinamización del terreno.

Para ello se ha elaborado un Índice de Cooperación, I_Coop, que mide la opinión de las empresas sobre la cooperación con diferentes actores. Se identifican estos actores, así como su importancia específica de cara a la construcción del índice global en la siguiente tabla:

Tabla 2. Relevancia de los actores para el fomento de la cooperación

\begin{tabular}{|l|c|r|r|r|r|r|c|}
\hline $\begin{array}{c}\text { Importancia } \\
\text { respecto } a \\
\text { I_Coop }\end{array}$ & \multicolumn{1}{|c|}{$1^{\circ}$} & \multicolumn{1}{c|}{$4^{\circ}$} & \multicolumn{1}{c|}{$5^{\circ}$} & \multicolumn{1}{c|}{$7^{\circ}$} & $6^{\circ}$ & $2^{\circ}$ & $3^{\circ}$ \\
\hline \multicolumn{1}{|c}{$I_{-C o o p}$} & Clientes & Proveedores & Competidores & Asociaciones & CCTT & $\begin{array}{c}\text { Cap. } \\
\text { Humano }\end{array}$ & FP \\
\hline Fundición & $38,76 \%$ & $10,73 \%$ & $9,10 \%$ & $4,02 \%$ & $5,01 \%$ & $16,78 \%$ & $15,59 \%$ \\
\hline $\begin{array}{l}\text { Estampación, } \\
\text { Forja }\end{array}$ & $37,09 \%$ & $11,14 \%$ & $9,42 \%$ & $4,16 \%$ & $4,70 \%$ & $17,47 \%$ & $15,86 \%$ \\
\hline Mecanizado & $38,97 \%$ & $11,50 \%$ & $8,28 \%$ & $2,54 \%$ & $2,79 \%$ & $20,53 \%$ & $15,37 \%$ \\
\hline $\begin{array}{l}\text { Calderería } \\
\text { Soldadura }\end{array}$ & $36,68 \%$ & $12,23 \%$ & $10,52 \%$ & $3,19 \%$ & $2,55 \%$ & $20,60 \%$ & $14,18 \%$ \\
\hline $\begin{array}{l}\text { Montaje y } \\
\text { Fabricación } \\
\text { de Equipos }\end{array}$ & $40,26 \%$ & $9,65 \%$ & $10,49 \%$ & $2,89 \%$ & $4,21 \%$ & $21,26 \%$ & $11,21 \%$ \\
\hline Tratamientos & $40,17 \%$ & $9,35 \%$ & $10,10 \%$ & $2,55 \%$ & $3,61 \%$ & $21,47 \%$ & $12,72 \%$ \\
\hline Plásticos & $35,53 \%$ & $12,88 \%$ & $10,84 \%$ & $4,35 \%$ & $4,98 \%$ & $12,38 \%$ & $19,00 \%$ \\
\hline Otros & $42,28 \%$ & $11,90 \%$ & $10,46 \%$ & $2,66 \%$ & $3,01 \%$ & $21,88 \%$ & $8,09 \%$ \\
\hline
\end{tabular}

Fuente: Elaboración Propia.

Destaca la importancia que adquieren los clientes para la cooperación global, no así las redes con proveedores. No se identifican diferencias reseñables en función de la actividad principal desarrollada por la organización. Las mayores diferencias en función de la actividad ejercida en la empresa, se hallan en relación con la vinculación a Centros de Formación Profesional, así como al Capital Humano. En relación con este factor, destaca como las empresas de actividad plástica, -ajenas a la cadena de valor territorial, aunque con importancia relativa dentro de la comarca- muestran el menor grado de apertura a 
las personas del entorno. Este hecho se puede atribuir a un know how escaso en actividades de transformación ajenos al metal.

El grado de cooperación viene delimitado, por la colaboración con los Centros de de Formación Profesional, y posteriormente por su vinculación con el Centro Tecnológico activo de la Comarca. Tanto la AED, como asociación empresarial colectiva, como el propio AZTERLAN se perciben como los actores relevantes para traccionar relaciones colaborativas en el terreno.

En relación con la Administración Pública Local, el 74\% de las empresas encuestadas consideran que estos agentes deben de implicarse para mejorar la posición del tejido productivo, y un $86 \%$ consideran que estas instituciones deben identificar proyectos en cooperación que mejoren el desempeño individual y colectivo.

\section{Conclusiones}

Esta investigación buscaba validar los actores pertenecientes a la red del Polo de Competitividad, con objeto de disponer de una herramienta que fomentase la competitividad del terreno, a partir de proyectos innovadores.

El análisis cuantitativo y cualitativo ha permitido ponderar la valoración sobre la fortaleza del tejido empresarial comarcal, siendo la cohesión interna uno de los pilares fundamentales del terreno. Si bien analizados individualmente el Centro Tecnológico AZTERLAN y la Asociación AED carecerían de valor, el análisis conjunto confirma la importancia que muestran los actores que conforman la red de cara a la dinamización del terreno, al contar con la confianza de las empresas.

De esta forma, se podría considerar como una herramienta de potencial interés para el desarrollo territorial la disposición de una red de actores como la aquí presentada que aunase el desempeño público, el conocimiento tecnológico, y el ejercicio productivo.

BiBLIOGRAFíA

ALBORS GARRIGÓS, J. and HIDALGO NUCHERA, A. "Relaciones de gobernanza e innovación en la cadena de valor: nuevos paradigmas de competividad," Revista Europea de Dirección y Economía de la Empresa (21:2), 2012, pp. 205-214.

CAÑIBANO, C. "El capital humano: factor de competitividad, innovación y crecimiento", Sexto congreso de economía de Navarra, 2005.

ERIKSSON, A. Regionala innovationssystem: från teori till genomförande, Sveriges tekniska attachéer, 2000.

ETZKOWITZ, H. "The Triple Helix: academy-industry-governement relations and the growth of neo-corporatist industrial policy in the US," Managing Technological Knowledge Transfer, EC Social Sciences COST A (3), 1997. 
EUSTAT, 2010: Tabla comparativa de ne Niveles Formativos. http://www. eustat.es/

MOHANNAK, K. "Technological Innovation in Japan, South Korea and Australia: A Linkage Policy Model”, 1998.

MOULAERT, F. and SEKIA, F. "Territorial innovation models: a critical survey," Regional studies (37:3), 2003, pp. 289-302.

Otero, B. "Made in Euskadi: Innovando en el sector de máquina-herramienta de la CAPV", 2010.

PCTI, 2012. "Plan de Ciencia Tecnología e Innovación 2015 del Gobierno Vasco". http://www.euskadinnova.net/es/innovacion-tecnologica/ambitosactuacion/pcti-2015/163.aspx

PUTNAM, R. D. Bowling alone: The collapse and revival of American community, Simon \& Schuster, 2001.

TÉLLEZ, T. and INOSCENCIA, B. Modelo de cadena de valor para el desarrollo rural: el caso del sector ovino en México y España", 2011. 